forward to learning the answers to these questions.

\section{Acknowledgments}

The authors are supported in part by NIH grants R01DK56366, P30DK56336, and R01ES09912.

1. Sturm, R. 2003. Increases in clinically severe obesity in the United States, 1986-2000. Arch. Intern. Med. 163:2146-2148.

2. National Institute of Diabetes \& Digestive \& Kidney Diseases. Phenotyping obesity for human genetic studies. http:/www.niddk.nih.gov/fund/ other/phenotyping/index.

3. Chagnon, Y.C., et al. 2003. The human obesity gene map: the 2002 update. Obes. Res. 11:313-367.

4. Redden, D.T., and Allison, D.B. 2003. Non-replication in genetic association studies of obesity and diabetes. J. Nutr. 133:3323-3326.

5. Rosmond, R. 2003. Association studies of genetic polymorphisms in central obesity: a critical review. Int. J. Obes. Relat. Metab. Disord. 27:1141-1151.

6. Hirschhorn, J.N., and Altshuler, D. 2002. Once and again - issues surrounding replication in genetic association studies. J. Clin. Endocrinol.
Metab. 87:4438-4441.

7. Allison, D.B., et al. 2003. Genetic influences on obesity. In Obesity: mechanisms and clinical management. R. Eckel, editor. Elsevier. New York, New York, USA. 31-74.

8. Segal, N., and Allison, D.B. 2002. Twins and virtual twins: bases of relative body weight revisited. Int. J. Obesity. 26:437-441.

9. Öhman, M., et al. 2000. Genome-wide scan of obesity in Finnish sibpairs reveals linkage to chromosome Xq24. J. Clin. Endocrinol. Metab. 85:3183-3190

10. Suviolahti, E., et al. 2003. The SLC6A14 gene shows evidence of association with obesity. J. Clin. Invest. 112:1762-1772. doi:10.1172/JCI200317491.

11. Sargent, P.A., Sharpley, A.L., Williams, C., Goodall, E.M., and Cowen, P.J. 1997. 5-HT2C receptor activation decreases appetite and body weight in obese subjects. Psychopharmacology (Berl.). 133:309-312.

12. Leibowitz, S.F., and Alexander, J.T. 1998. Hypothalamic serotonin in control of eating behavior, meal size, and body weight. Biol. Psychiatry. 44:851-864.

13. Rankinen, T., and Tiwari, H.K. 2003. Genome scans for human nutritional traits. What have we learned? Nutrition. In press.

14. Hager, J., et al. 1998. A genome-wide scan for human obesity genes reveals a major susceptibility locus on chromosome 10. Nat Genet. 20:304-308.
15. Price, R.A., Li, W.D., and Kilker, R. 2002. An X chromosome scan reveals a locus for fat distribution in chromosome region Xp21-22. Diabetes. 51:1989-1991.

16. Stone, S., et al. 2002. A major predisposition locus for severe obesity, at 4p15-p14. Am.J. Hum Genet. 70:1459-1468.

17. Lander, E., and Kruglyak, L. 1995. Genetic dissection of complex traits: guidelines for interpreting and reporting linkage results. Nat. Genet. 11:241-247.

18. Page, G.P., George, V., Go, R.C., Page, P.Z., and Allison, D.B. 2003. "Are we there yet?": Deciding when one has demonstrated specific genetic causation in complex diseases and quantitative traits. Am. J. Hum. Genet. 73:711-719.

19. Cardon, L.R., and Palmer, L.J. 2003. Population stratification and spurious allelic association. Lancet. 361:598-604.

20. Silventoinen, K., Kaprio, J., Lahelma, E., Viken R.J., and Rose, R.J. 2003. Assortative mating by body height and BMI: Finnish twins and their spouses. Am. J. Human Biol. 15:620-627.

21. Allison, D.B., et al. 2002. Bias in estimates of quantitative-trait-locus effect in genome scans: demonstration of the phenomenon and a method-of-moments procedure for reducing bias. Am. J. Hum. Genet. 70:575-585.

22. Boerwinkle, E., Hixson, J.E., and Hanis, C.L. 2000 Peeking under the peaks: following up genomewide linkage analyses. Circulation. 102:1877-1888.

\title{
Genes required for B cell development
}

\author{
Mary Ellen Conley 1,2 \\ ${ }^{1}$ Department of Immunology, St. Jude Children's Research Hospital, Memphis, \\ Tennessee, USA \\ ${ }^{2}$ Department of Pediatrics, University of Tennessee College of Medicine, Memphis, \\ Tennessee, USA
}

\begin{abstract}
Mutations in a variety of genes can cause congenital agammaglobulinemia and a failure of B cell development. The currently known genes encode components of the pre-B cell receptor or proteins that are activated by cross-linking of the pre-B cell receptor. Defects in these genes result in a block in $B$ cell differentiation at the pro-B to pre-B cell transition. A patient with a translocation involving a previously unknown gene, $L R R C 8$, demonstrated a block at exactly the same point in B cell differentiation (see the related article beginning on page 1707). It will be interesting to determine whether the protein encoded by this gene interacts with the pre-B cell receptor signal transduction pathway or is involved in a new pathway.
\end{abstract}

J. Clin. Invest. 112:1636-1638 (2003). doi:10.1172/JCI200320408.
B cell maturation proceeds through a series of stages that can be defined by the rearrangement status of the $\mathrm{Ig}$ genes, the expression of cell surface markers, and the location of the cells within the bone marrow, the spleen, or the lymph nodes (1-3). Patients with defects in early B cell maturation usually develop recurrent infections, caused by encapsulated bacteria, in the first 2 years of life, and most are recog- nized to have immunodeficiency when they are hospitalized for a dramatic infection at less than 3 years of age (4). Approximately $80 \%$ of patients with the early onset of recurrent infections, hypogammaglobulinemia, and markedly reduced or absent $B$ cells have X-linked agammaglobulinemia (XLA) (5). This disorder is caused by mutations in a hematopoietic-specific cytoplasmic tyrosine kinase, Btk $(6,7)$. Btk is expressed in myeloid cells, in platelets, and at all stages of B cell development except plasma cells (8-10); however, it is important to note that patients with XLA have absent or markedly reduced numbers of $B$ cells but do not have any clinical abnormalities in myeloid cells or in platelet number or function.

The earliest point in B cell differentiation at which Btk is required coincides with expression of the pre-B cell receptor at the pro- $\mathrm{B}$ to pre-B cell transition $(11,12)$. Therefore, it is not surprising that defects in components of the pre-B cell receptor account for an additional $7-10 \%$ of patients with congenital agammaglobulinemia. The majority of these patients have mutations in the constant region of $\mu$ heavy chain Ig (13), but a small number have defects in $\lambda 5$ (14), which is part of the surrogate light chain, or in $\operatorname{Ig} \alpha(15)$, a transmembrane protein that binds to 


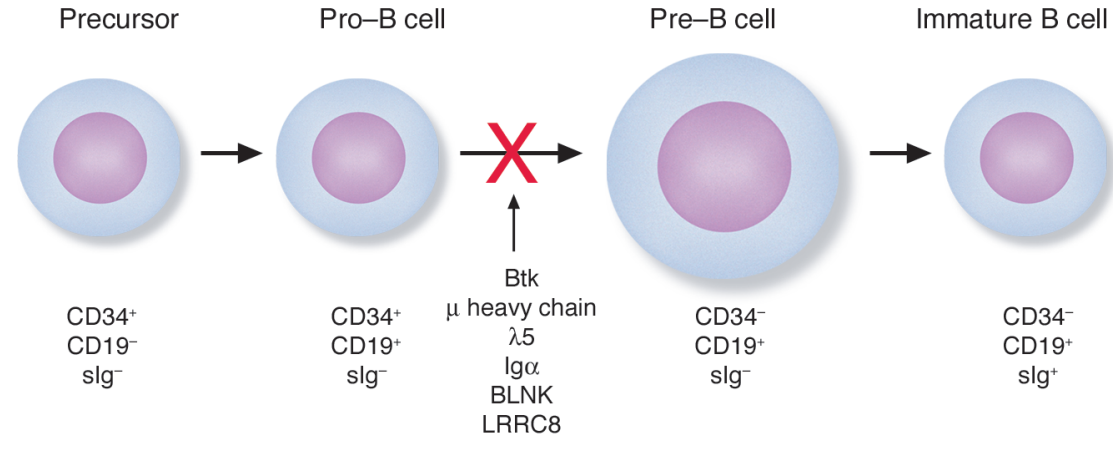

Figure 1

Early stages of B cell differentiation can be identified by the status of the I $g$ genes and by the cell surface markers CD34, CD19, and surface Ig (slg). In stem cells and common lymphoid precursors, the Ig genes are in germ-line configuration. These cells express CD34 but not CD19 or slg. In pro-B cells, cells committed to the B cell lineage, the first step in $\mu$ heavy chain gene rearrangement, D-to-J rearrangement, is occurring. All of the components of the pre-B cell receptor except $\mu$ heavy chain (the surrogate light chain proteins $\lambda 5$ and VpreB, and the proteins that constitute the pre-B cell receptor signal transduction module, Ig $\alpha$ and $\operatorname{Ig} \beta$ ) can be found in the cytoplasm of these cells. Pro-B cells are positive for CD34 and CD19, but they are negative for slg. Once an effective, in-frame VDJ heavy chain rearrangement has occurred, and the resulting $\mu$ heavy chain can bind to the surrogate light chain, the pre-B cell receptor can be displayed on the cell surface, and the cells become pre-B cells. Pre-B cells have a completely rearranged $\mu$ heavy chain gene, but the light chain genes are in germ-line configuration. These cells express cytoplasmic $\mu$ heavy chain and cell surface CD19, but they are negative for CD34 and slg. After successful rearrangement of a light chain gene, the conventional $\mathrm{B}$-cell receptor can be expressed on the cell surface, and the cell becomes an immature B cell that is positive for CD19 and slg and negative for CD34.

$\mu$ heavy chain and acts as part of the signal transduction module. Expression of these proteins is limited to the $B$ cell lineage. Downstream targets of activation through the pre-B cell or B cell receptor complex include Btk and $\mathrm{B}$ cell linker (BLNK), a scaffold protein that binds Btk, PLC $\gamma 2$, Grb2, Vav, and Nck. Two patients with agammaglobulinemia and defects in BLNK have been identified (ref. 16 and unpublished results). Patients with defects in components of the pre- $\mathrm{B}$ cell receptor, Btk, or BLNK all have a block in $\mathrm{B}$ cell differentiation at the pro- $\mathrm{B}$ to pre-B cell transition (17) (Figure 1).

\footnotetext{
Figure 2

(a) Wild-type LRRC8 is an 810-amino acid protein with four transmembrane domains. Both the amino-terminal and carboxy-terminal ends of this protein are extracellular. The carboxy-terminal end contains nine leucinerich repeats. (b) The mutant LRRC8, produced as a consequence of a translocation that transected the gene between exon 1 and exon 2, lacks the last two-and-a-half leucinerich repeats and contains 35 amino acids derived from the intron 1 sequence.
}

The remaining $10-15 \%$ of patients with the early onset of infections, agammaglobulinemia, and absent $B$ cells represent a heterogeneous group. Some of these patients have myelodys- plasia, with hypogammaglobulinemia and reduced numbers of $B$ cells being the first sign of their disease (18). Some patients have subtle $\mathrm{T}$ cell defects as well as more severe $\mathrm{B}$ cell defects; some have morphologic defects including failure to thrive, microcephaly, and/or abnormalities of the hands and feet (19-22); and some are clinically indistinguishable from patients with defects in $\mu$ heavy chain. A variety of approaches can be taken to identify the genes that are abnormal in these patients. Murine models of immunodeficiency resulting in decreased or absent B cells can provide some clues (23), but it is important to remember that mutations in the same gene may result in different phenotypes in mice and humans (17). Proteins belonging to the signal transduction pathways activated by cross-linking of the pre-B cell receptor are attractive candidates for analysis, particularly when the expression of the gene of interest is limited to hematopoietic cells. Careful evaluation of an unusual patient can be one of the most rewarding approaches.

In this issue of the JCI, Sawada et al. describe a 17-year-old girl with unusual facial features, agammaglobulinemia, and markedly reduced or absent $B$ cells (24). Karyotype studies revealed a

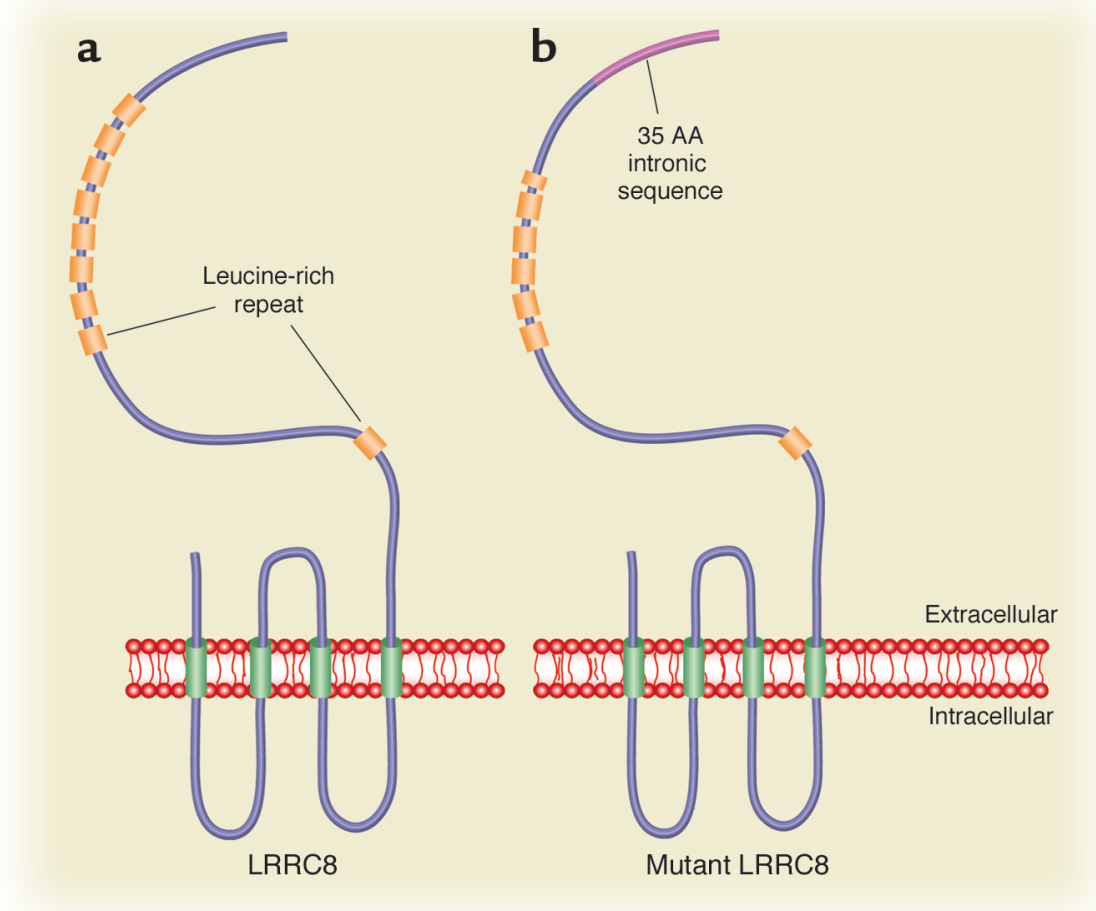


balanced translocation between chromosomes 9 and 20, resulting in the truncation of a novel gene, leucine-rich repeat-containing 8 (LRRC8), normally encoded on the long arm of chromosome 9. This unusual gene consists of only two exons, which encode an 810 -amino acid protein with four transmembrane domains and an extracellular carboxy-terminal domain with nine leucine-rich repeats (Figure 2). The translocation occurred between the first and second exons and resulted in the loss of the last two-and-a-half leucine-rich regions and the addition of 35 amino acids from the intronic sequence. Using immunofluorescence staining and RT-PCR, the authors suggest that the gene is expressed in brain, heart, lung, liver, and kidney as well as $\mathrm{T}$ cells and B-lineage cells.

Based on the observation that there were no abnormalities in the other allele of LRRC8, and that protein from the normal and abnormal alleles were expressed in white blood cells from the patient, the authors interpret their results as indicating that the mutation had a dominant-suppressor effect on B cell development (24). It is interesting to speculate on how this dominant effect might occur and whether there might be other patients with agammaglobulinemia due to similar mutations. Several mechanisms can explain an autosomal dominant effect. For example, if the last two leucine-rich domains function as self-inhibitory domains, the loss of those domains might result in a constitutively active protein; or, if the protein is part of a multimeric complex that includes more than one copy of the LRRC8 protein, one abnormal allele could alter the function of a majority of complexes. Occasionally, an abnormal protein can change the stability or location of a binding partner and thereby inhibit the ability of the binding protein to function. By reconstituting lethally irradiated mice with bone marrow that had been transfected with a retroviral vector expressing the mutant protein, the authors showed that the mutant protein caused a block at the pro- $\mathrm{B}$ to pre- $\mathrm{B}$ cell transition (Figure 1). This brings up the possibility that LRRC8 might interact with the pre-B cell receptor in some way. Alternatively, the 35 amino acids derived from the intronic sequence at the carboxy-terminal portion of the mutant LRRC8 might confer on the protein a toxic function similar to that of the polyglutamine tract in trinucleotide repeat disorders.

At this time it is difficult to say whether there are likely to be other patients with congenital immunodeficiency due to defects in LRRC8. The authors note that the gene is broadly expressed and that the gene product can be found on the surface of $\mathrm{T}$ cells. A detailed evaluation of $T$ cell function might result in the detection of subtle findings that would allow one to identify similar patients. The authors also describe their patient as having mildly dysmorphic facial features. Because many otherwise normal people have mildly dysmorphic facial features, it is not clear at this time whether the unusual facial features of the individual examined by Sawada et al. (24) are secondary to the defect in LRRC8 or part of her familial inheritance. Finding an abnormal gene in a patient with immunodeficiency is only the first exciting step. The next steps should tell us how LRRC8 functions in the normal immune system.

1. Rajewsky, K. 1996. Clonal selection and learning in the antibody system. Nature. 381:751-758.

2. Conley, M.E., and Cooper, M.D. 1998. Genetic basis of abnormal B cell development. Curr. Opin. Immunol. 10:399-406.

3. Hardy, R.R. 2003. B-cell commitment: deciding on the players. Curr. Opin. Immunol. 15:158-165.

4. Conley, M.E., and Howard, V. 2002. Clinical find ings leading to the diagnosis of $\mathrm{X}$-linked agammaglobulinemia. J. Pediatr. 141:566-571.

5. Conley, M.E., Mathias, D., Treadaway, J., Minegishi, Y., and Rohrer, J. 1998. Mutations in Btk in patients with presumed $X$-linked agammaglobulinemia. Am. J. Hum. Genet. 62:1034-1043.

6. Vetrie, D., et al. 1993. The gene involved in Xlinked agammaglobulinemia is a member of the src family of protein-tyrosine kinases. Nature. 361:226-233.

7. Tsukada, S., et al. 1993. Deficient expression of a B cell cytoplasmic tyrosine kinase in human Xlinked agammaglobulinemia. Cell. 72:279-290.

8. Smith, C.I.E., et al. 1994. Expression of Bruton's agammaglobulinemia tyrosine kinase gene, BTK is selectively down-regulated in T lymphocytes and plasma cells. J. Immunol. 152:557-565.

9. Genevier, H.C., et al. 1994. Expression of Bruton's tyrosine kinase protein within the B cell lineage. Eur. J. Immunol. 24:3100-3105.

10. Quek, L.S., Bolen, J., and Watson, S.P. 1998. A role for Bruton's tyrosine kinase (Btk) in platelet activation by collagen. Curr. Biol. 8:1137-1140.

11. Campana, D., Farrant, J., Inamdar, N., Webster, A.D.B., and Janossy, G. 1990. Phenotypic features and proliferative activity of B cell progenitors in $\mathrm{X}$-linked agammaglobulinemia. J. Immunol. 145:1675-1680.

12. Nomura, K., et al. 2000. Genetic defect in human $\mathrm{X}$-linked agammaglobulinemia impedes a maturational evolution of pro-B cells into a later stage of pre-B cells in the B-cell differentiation pathway. Blood. 96:610-617.

13. Granados, E.L., et al. 2002. Clinical and molecular analysis of patients with defects in $\mu$ heavy chain gene. J. Clin. Invest. 110:1029-1035. doi:10.1172/JCI200215658.

14. Minegishi, Y., et al. 1998. Mutations in the human $\lambda 5 / 14.1$ gene result in B cell deficiency and agammaglobulinemia. J. Exp. Med. 187:71-77.

15. Minegishi, Y., et al. 1999. Mutations in Igo (CD79a) result in a complete block in B cell development. J. Clin. Invest. 104:1115-1121.

16. Minegishi, Y., et al. 1999. An essential role for BLNK in human B cell development. Science. 286:1954-1957.

17. Conley, M.E., Rohrer, J., Rapalus, L., Boylin, E.C., and Minegishi, Y. 2000. Defects in early B-cell development: comparing the consequences of abnormalities in pre-BCR signaling in the human and the mouse. Immunol. Rev. 178:75-90.

18. Srivannaboon, K., Conley, M.E., Coustan-Smith, E., and Wang, W.C. 2001. Hypogammaglobulinemia and reduced numbers of $\mathrm{B}$ cells in children with myelodysplastic syndrome. J. Pediatr. Hema tol. Oncol. 23:122-125.

19. Revy, P., et al. 2000. A syndrome involving intrauterine growth retardation, microcephaly, cerebellar hypoplasia, B lymphocyte deficiency, and progressive pancytopenia. Pediatrics. 105:E39.

20. Verloes, A., Dresse, M.F., Keutgen, H., Asplund, C., and Smith, C.I. 2001. Microphthalmia, facial anomalies, microcephaly, thumb and hallux hypoplasia, and agammaglobulinemia. Am. J. Med. Genet. 101:209-212.

21. Hoffman, H.M., Bastian, J.F., and Bird, L.M. 2001 Humoral immunodeficiency with facial dysmorphology and limb anomalies: a new syndrome. Clin. Dysmorphol. 10:1-8.

22. Adderson, E.E., et al. 2000. Growth failure, intracranial calcifications, acquired pancytopenia, and unusual humoral immunodeficiency: a genetic syndrome? Am. J. Med. Genet. 95:17-20.

23. Loffert, D., et al. 1994. Early B-cell development in the mouse: insights from mutations introduced by gene targeting. Immunol. Rev. 137:135-153.

24. Sawada, A., et al. 2003. A congenital mutation of the novel gene LRRC8 causes agammaglobulinemia in humans. J. Clin. Invest. 112:1707-1713. doi:10.1172/JCI200318937. 